\title{
Multisystemic inflammatory syndrome post-COVID-19: A new emerging entity
}

\author{
Vijay B Sonawane', Veeranna Kotrashetti ${ }^{2}$, Kapil Bainade', V Trisha ${ }^{3}$, Saili Bunde ${ }^{3}$, Saniya Sroa ${ }^{3}$

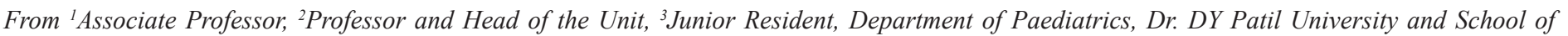 \\ Medicine, Navi Mumbai, Maharashtra, India
}

\begin{abstract}
Multisystem inflammatory syndrome in children (MISC-C), also known as the pediatric inflammatory multisystem syndrome, is a new dangerous childhood disease that may be associated with COVID disease. Signs and symptoms depend on the body part involved. We report a case of complicated post-COVID MIS-C in a 5-year-old child who presented with fever, vomiting, pain abdomen for 6 days, and rash for 2 days. Since the patient had complaints of high-grade fever for more than 5 days, rash, non-purulent conjunctivitis, cracked lips with cardiac and respiratory, and gastrointestinal involvement with positive COVID antibodies, and raised CRP; she was diagnosed to have the MIS-C post-COVID. The patient was started on oral aspirin and oral prednisolone for 2 weeks and discharged with proper advice.
\end{abstract}

Key words: Multisystem inflammatory syndrome, Pediatric inflammatory multisystem syndrome, Post-COVID

$\mathrm{M}$ ultisystem inflammatory syndrome in children (MIS-C) is a serious, though rare condition in which the body's own immune system overreacts to a stimulus, resulting in inflammation of multiple organ systems throughout the body. It can affect the heart, blood vessels, lungs, and other respiratory organs, kidneys, skin, eyes, and nervous system, and it can lead to organ dysfunction and even organ failure. The involvement of more than 2 organs is required to be kept as a requisite definition. MIS-C shows similarities to other known inflammatory disorders, including Kawasaki disease which is characterized by the inflammation in the walls of blood vessels in the body. Common features between Kawasaki and MIS-C are high-grade fever, rashes, conjunctivitis, and chapped lips. It may also show similarity with toxic shock syndrome which is characterized by high-grade fever, nausea, vomiting, diarrhea, or organ dysfunction. In April of 2020, reports from the United Kingdom documented a presentation in children similar to incomplete Kawasaki disease (KD) or toxic shock syndrome $[1,2]$. Since then, there have been reports of similarly affected children in other parts of the world [3]. We report the case of a 5-year-old child who presented with serositis and hypotension.

\section{CASE REPORT}

A 5-year-old female child presented to our department with complaints of fever, vomiting, pain abdomen for 6 days, and rash for

\section{Access this article online}

Received - 02 May 2021

Initial Review - 18 May 2021

Accepted - 11 June 2021

DOI: $10.32677 /$ IJCR.2021.v07.i06.011
2 days. Fever was undocumented high-grade which was associated with chills and rigors and reduced after taking medications. Vomiting was non-projectile, containing food particles, nonbilious, non-blood tinged with 4-5 episodes per day. Pain in the abdomen was diffuse, not associated with any aggravating or relieving factors. Rash was erythematous, initially found over the trunk and upper limb but gradually progressed to the entire body which was also associated with itching all over the body.

On examination, the patient was febrile with a temperature of $101 \mathrm{~F}$ in the axilla, heart rate was $100 / \mathrm{min}$, respiratory rate was $28 / \mathrm{min}, \mathrm{SpO}_{2}$ was $97 \%$ on RA, and blood pressure was $94 / 50 \mathrm{~mm} \mathrm{Hg}\left(50^{\text {th }}\right.$ percentile $)$. Head-to-toe examination revealed mild periorbital and lip edema, erythematous papular rash all over the body, conjunctivitis, and cracked lips. On systemic examination, the abdomen was distended, tenderness was present, and shifting dullness was present on percussion.

All relevant investigations were done. Blood investigation showed hemoglobin of $9.5 \mathrm{~g} / \mathrm{dl}$, white blood cells (WBCs) count of $4.9 \times 10^{9} / \mathrm{L}$, and platelet count of $161,000 / \mathrm{mm}^{3}$. C-reactive protein (CRP) was grossly elevated with a value of $206.03 \mathrm{mg} / \mathrm{dl}$. The malarial antigen test was negative. Widal test and IgM for typhoid were negative. Serum electrolytes were sodium - 134/ potassium $-3.8 /$ chloride $-105 \mathrm{mEq} / \mathrm{L}$. Chest X-ray showed the features of ARDS and cardiomegaly (Fig. 1). Ultrasound (USG) abdomen was done in view of pain abdomen which showed mildto-moderate ascites, multiple lymph nodes were noted in the right iliac fossa and mesentery, the largest measuring $1.6 \times 1.2 \mathrm{~cm}$ in the right iliac fossa.

Correspondence to: Dr. V Trisha, 501 - D, Priyadarshini Block, Dr. DY Patil Girls Hostel, Dr. DY Patil Medical College, Sector-5, Nerul, Navi Mumbai, Maharashtra, India. E-mail: vtrishajss@gmail.com

(C) 2021 Creative Commons Attribution-NonCommercial 4.0 International License (CC BY-NC-ND 4.0). 


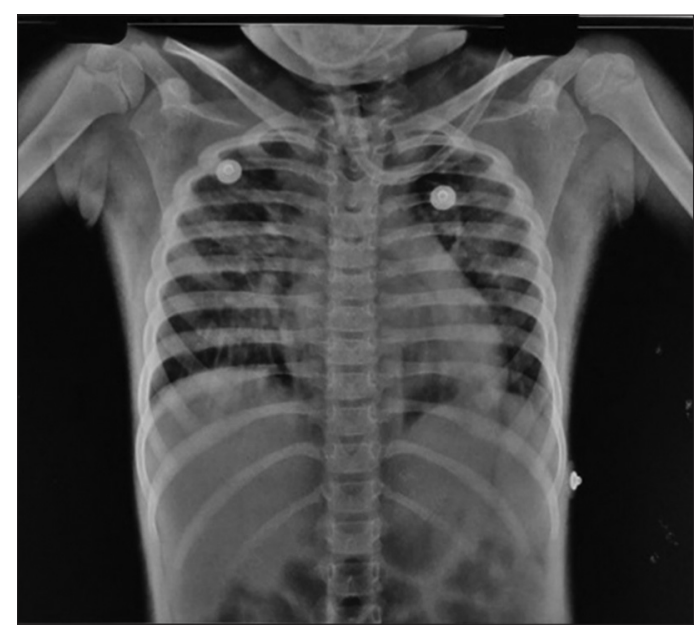

Figure 1: Chest X-ray showing features of ARDS and cardiomegaly

On day 3 of admission, the blood pressure was less than $50^{\text {th }}$ percentile, capillary refilling time was delayed, and peripheries were cold, so IV bolus was given. 2D ECHO was done which showed bilateral pleural effusion with a thin rim of pericardial effusion with serositis with mild mitral regurgitation and trivial tricuspid regurgitation with an ejection fraction of $63 \%$. $\mathrm{N}$ terminal pro B-type natriuretic peptide (NT-proBNP) was done which was normal. Since CRP $(236 \mathrm{mg} / \mathrm{L})$, ferritin $(846 \mathrm{ng} / \mathrm{ml})$, and D-Dimer $(>4000 \mathrm{ng} / \mathrm{ml})$ were markedly elevated, the patient was suspected to have MISC.

The patient was started on noradrenaline, low-molecularweight heparin $(35 \mathrm{mg} / \mathrm{kg} / \mathrm{day})$ in view of raised D-Dimer, methylprednisolone $(2 \mathrm{mg} / \mathrm{kg} /$ day $)$, and prophylactic antibiotics. Repeat 2D ECHO showed decreased pericardial effusion. Inotropes were tapered, fluids were gradually reduced, and oral feed was started. Since the patient had complaints of high-grade fever for more than 5 days, rash, non-purulent conjunctivitis, cracked lips with cardiac and respiratory, and gastrointestinal involvement with positive COVID antibodies, and raised CRP; she was diagnosed to have the MIC-C, post-COVID.

Repeat investigations showed reduced CRP and D-dimer; hence, the patient was started on oral aspirin and oral prednisolone at the time of discharge which was tapered and stopped in the next 2 weeks. The child was accepting feeds orally, passing urine and stool adequately, and hemodynamically stable. The patient was discharged with proper advice to continue tablet furosemide till the next 2D ECHO, no live vaccines till 11 months, and no NSAID till aspirin is continued. A follow-up of the patient was scheduled after 2 weeks and 6 weeks for 2D ECHO.

\section{DISCUSSION}

Multisystem inflammatory syndrome is a new pediatric disease associated with coronavirus that is dangerous and potentially lethal. MIS-C is also known as a pediatric MIS (PMIS), pediatric inflammatory multisystem syndrome temporally associated with SARS-CoV-2 (PIMS-TS), pediatric hyperinflammatory syndrome, or pediatric hyperinflammatory shock. With prompt recognition and medical attention, most children will survive but the long-term outcomes from this condition are presently unknown. Further studies and longer surveillance of patients with MIS-C are required to improve our diagnostic, treatment, and surveillance criteria.

The pathogenesis of MIS-C is not well understood, and it is thought to be a postinfectious immune-mediated phenomenon, as seen from a lag of 2-4 weeks between the occurrence of peak incidence of COVID-19 cases in communities and the recognition of MIS-C [4].

All affected children have a persistent fever. The first symptoms often include acute abdominal pain with diarrhea or vomiting. Myalgia and general tiredness are frequent, and low blood pressure is also common [5]. Other abnormal findings can be pink eye, rashes, enlarged lymph nodes, swollen hands and feet, and "strawberry tongue." Various mental disturbances are possible [6]. Clinical complications can include damage to the heart muscle, respiratory distress, acute kidney injury, and increased blood coagulation [7]. Coronary artery abnormalities can develop ranging from dilatation to aneurysms [5]. According to the present classification by the $\mathrm{WHO}$, any child presenting with persistent fever, inflammation (neutrophilia, elevated CRP, and lymphopenia), and evidence of single or multiorgan dysfunction with the exclusion of other infective cause and evidence of COVID-19 infection is labeled as MIS-C [8].

According to the World Health Organization, management of MIS should involve multidisciplinary care with intensive care, pediatric cardiology, infectious disease, and rheumatology specialists [8]. A small proportion of children go on to develop severe acute COVID-19 disease and require hospitalization because of respiratory compromise or complications of SARSCoV-2 infection such as MIS-C. A Centers for Disease Control and Prevention (CDC) study in August 2020 of 576 children hospitalized with SARS-CoV-2 infection reported a lower hospitalization rate compared with adults ( 8 vs. 165 per 100000 ); however, one in three hospitalized children required admission to intensive care units, a rate similar to adults [4].

As there are increasing cases of COVID-19 in India, the pediatrician should be acutely aware of this new clinical syndrome related to SARS-CoV-2 infection that overlaps with signs and symptoms of Kawasaki disease. Clinicians should operate with an increased index of suspicion and follow the case definition proposed by the WHO and CDC to identify this syndrome early in the course of illness. Prompt identification with the employment of appropriate therapies is the key to favorable outcomes [9]. MIS-C is rare but the potential long-term sequelae from this disease are currently unknown. As we are in the midst of understanding these novel manifestations of COVID-19 in children, reporting is essential for clinicians for the timely initiation of appropriate management.

\section{CONCLUSION}

MIS-C is a dangerous systemic infection characterized by extreme inflammation, fever, abdominal symptoms, conjunctivitis, and rash. Children will typically show signs/symptoms of MIS-C 
3-4 weeks after COVID-19 infection and many will progress rapidly into shock and cardiorespiratory failure. Families should seek immediate medical care as children with this condition decompensate quickly and most children will need management in an intensive care unit. Overall, children will survive this hyperinflammatory condition with the administration of IVIG, steroids, a multidisciplinary team of health-care providers, and in some cases immunomodulatory agents.

\section{REFERENCES}

1. Riphagen S, Gomez X, Gonzalez-Martinez C, Wilkinson N, Theocharis P. Hyperinflammatory shock in children during COVID-19 pandemic. Lancet 2020;395:1607.

2. Paediatric Intensive Care Society (PICS) Statement: Increased Number of Reported Cases of Novel Presentation of Multi System Inflammatory Disease. Available from: https://www.picsociety.uk/wp-content/uploads/2020/04/ PICS-statement-re-novel-KD-C19-presentation-v2-27042020.pdf. [Last accessed on 2020 May 15].

3. Health Alert Network (HAN): Multisystem Inflammatory Syndrome in Children (MIS-C) Associated with Coronavirus Disease 2019 (COVID-19). Available from: https://www.emergency.cdc.gov/han/2020/han00432.asp. [Last accessed on 2020 May 15].

4. Centers for Disease Control and Prevention. 2020. Available from: https:// www.cdc.gov/mis-c/hcp. [Last accessed on $2020 \mathrm{Jul}$ 31].

5. Ahmed M, Advani S, Moreira A, Zoretic S, Martinez J, Chorath K, et al.
Multisystem inflammatory syndrome in children: A systematic review. EClinicalMedicine 2020;26:100527.

6. Henderson LA, Canna SW, Friedman KG, Gorelik M, Lapidus SK, Bassiri $\mathrm{H}$, et al. American college of rheumatology clinical guidance for pediatric patients with multisystem inflammatory syndrome in children (MIS-C) associated with SARS-CoV-2 and hyperinflammation in COVID19: Version 1. Arthritis Rheumatol 2020;72:1791-805.

7. Aronoff SC, Hall A, Del Vecchio MT. The natural history of SARSCov-2 related multisystem inflammatory syndrome in children (MIS-C): A systematic review. J Pediatr Infect Dis Soc 2020;9:746-51.

8. World Health Organization. Scientific Brief. Multisystem Inflammatory Syndrome in Children and Adolescents Temporally Related to COVID19. Geneva: World Health Organization; 2020. Available from: https:// www.who.int/news-room/commentaries/detail/multisystem-inflammatorysyndrome-in-children-and-adolescents-with-covid-19. [Last accessed on 2020 Jul 31].

9. Whittaker E, Bamford A, Kenny J, Kaforou M, Jones CE, Shah P, et al. Clinical characteristics of 58 children with a pediatric inflammatory multisystem syndrome temporally associated with SARS-CoV-2. JAMA 2020;324:259-69.

Funding: None; Conflicts of Interest: None Stated.

How to cite this article: Sonawane VB, Kotrashetti V, Bainade K, Trisha V, Bunde S, Sroa S. Multisystemic inflammatory syndrome post-COVID-19: A new emerging entity. Indian J Case Reports. 2021;7(6):254-256. 